OPEN ACCESS

Edited by:

Yusuke Moriguchi,

Kyoto University, Japan

Reviewed by:

M. Rosario Rueda

University of Granada, Spain

Sabine Heim

Rutgers University, USA

*Correspondence:

Elisabet Suades-González elisabet.suades@isglobal.org

Specialty section: This article was submitted to Developmental Psychology, a section of the journal

Frontiers in Psychology

Received: 23 December 2016 Accepted: 12 April 2017

Published: 16 May 2017

Citation:

Suades-González E, Forns J, García-Esteban R, López-Vicente M,

Esnaola M, Álvarez-Pedrerol $M$,

Julvez J, Cáceres A, Basagaña X, López-Sala A and Sunyer J (2017) A Longitudinal Study on Attention Development in Primary School

Children with and withou Teacher-Reported Symptoms of ADHD. Front. Psychol. 8:655. doi: 10.3389/fpsyg.2017.00655

\section{A Longitudinal Study on Attention Development in Primary School Children with and without Teacher-Reported Symptoms of ADHD}

\author{
Elisabet Suades-González 1,2,3,4*, Joan Forns 1,2,4, Raquel García-Esteban 1,2,4, \\ Mónica López-Vicente ${ }^{1,2,4,5}$, Mikel Esnaola ${ }^{1,2,4}$, Mar Álvarez-Pedrerol 1,2,4, \\ Jordi Julvez ${ }^{1,2,4,5,6}$, Alejandro Cáceres ${ }^{1,4}$, Xavier Basagaña ${ }^{1,2,4}$, Anna López-Sala ${ }^{3}$ and \\ Jordi Sunyer ${ }^{1,2,4,5}$ \\ 'Center for Research in Environmental Epidemiology, Barcelona Institute for Global Health, Barcelona, Spain, ${ }^{2}$ Department of \\ Experimental and Health Sciences, Pompeu Fabra University, Barcelona, Spain, ${ }^{3}$ Learning Disabilities Unit (UTAE), \\ Neuropediatrics Department, Hospital Sant Joan de Déu, University of Barcelona, Barcelona, Spain, ${ }^{4}$ CIBER Epidemiología y \\ Salud Pública, Barcelona, Spain, ${ }^{5}$ Hospital del Mar Medical Research Institute, Barcelona, Spain, ${ }^{6}$ Environmental Health \\ Department, Harvard T.H. Chan School of Public Health, Boston, MA, USA
}

Background: Prospective longitudinal studies are essential in characterizing cognitive trajectories, yet few of them have been reported on the development of attention processes in children. We aimed to explore attention development in normal children and children with attention deficit and hyperactivity disorder (ADHD) symptoms in a repeated measures design using the attention network test (ANT).

Methods: The population sample included 2,835 children (49.6\% girls) aged $7-11$ years from 39 schools in Barcelona (Catalonia, Spain) who performed the ANT four times from January 2012 to March 2013. According to teacher ratings, 10.5\% of the children presented ADHD symptoms. We performed multilevel mixed-effects linear regression models, adjusting for school and individual, to test the effects of age-related growth on the ANT networks: alerting, orienting and executive attention, and three measurements related to attentiveness: median of hit reaction time (HRT), hit reaction time standard error (HRT-SE) and variability.

Results: We observed age-related growth in all the outcomes, except orienting. The curves were steeper at the younger groups, although for alertness the improvement was further at the oldest ages. Gender and ADHD symptoms interacted with age in executive attention, HRT and variability. Girls performed better in executive attention at young ages although boys reached females at around 10 years of age. For HRT, males showed faster HRT. However, girls had a more pronounced improvement and reached the levels of boys at age 11. Children with ADHD symptoms had significant differences in executive attention, HRT and variability compared to children without ADHD symptoms. 
Conclusions: We detected an ongoing development of some aspects of attention in primary school children, differentiating patterns by gender and ADHD symptoms. Our findings support the ANT for assessing attention processes in children in large epidemiological studies.

Keywords: attention network test, attention, children, neurodevelopment, multilevel analysis, population study, longitudinal study

\section{INTRODUCTION}

Attention is a complex cognitive function involving different processes like selectively attending to specific stimuli, focusing for prolonged periods, or regulating and monitoring of actions (Anderson, 2002). It is a basic function that precedes higherlevel cognitive abilities (e.g., executive functions or memory). Furthermore, attention underlies our awareness of the world and the voluntary regulation of our thoughts and feelings (Posner and Rothbart, 2007). The evaluation of attention in children is important because of its implications on learning, academic achievement and social functioning (Spira and Fischel, 2005).

Posner and Petersen were the first to propose a framework that divides attention into three functionally and anatomically separate networks: alerting, orienting and executive attention (Posner and Petersen, 1990; Fan et al., 2005). Alerting is the ability to produce and maintain optimal vigilance and performance during tasks (Petersen and Posner, 2012); the brain areas implicated are locus coeruleus, right frontal and parietal cortex. Orienting involves shifting attention to endogenous or exogenous cues (Corbetta et al., 1998; Posner et al., 2006); it involves parietal sites and frontal eye fields. Executive attention involves detecting and resolving conflict among responses, error detection and response inhibition (Bush et al., 2000); anterior cingulate cortex and prefrontal areas are involved in this network.

Following Posner and Petersen's model, Fan et al. developed the attention network test (ANT) to assess the three attentional networks (Fan et al., 2002). The ANT combines the flanker task (Eriksen and Eriksen, 1974), a widely used measure of executive control processes, with the use of cues entailing just alertness or alertness plus orienting information.

Rueda et al. adapted the adult ANT with a format and design more attractive for use with children from 4 years of age (Rueda et al., 2004). It is a rapid and easy-to-apply computerized test that has been validated as a tool for measuring attention processes in large-scale epidemiological studies (Forns et al., 2014) in which efficiency, precision and objectivity of data collection from using a computerized format is a major benefit.

Differences between age-group means in children, adolescents and adults have been encountered in attention processes using the ANT according to previous cross-sectional studies (Mezzacappa, 2004; Rueda et al., 2004; Gupta and Kar, 2009; Federico et al., 2017). Attention starts to develop early in infancy (e.g., exogenous alertness, orienting to external cues). Then, by the end of the first year of life, a more voluntarily controlled attention emerges. During childhood, the three networks continue developing and showing improvement in the endogenous sustained attention, the reorienting of attention, and the inhibitory control. Furthermore, the executive attention network, which depends on the maturation of the prefrontal cortex, shows a longer development into adolescence (Welsh and Pennington, 1988; Rueda et al., 2004, 2015; Konrad et al., 2005; Romine and Reynolds, 2005; Amso and Johnson, 2006). This is in relation to the many neurophysiological changes that occur in the brain (proliferation, migration, differentiation, synaptogenesis, myelination, and apoptosis) from the embryonic period through adolescence (Rice and Barone, 2000). In addition, there are genetic and environmental contributions to these changes on the developing brain, and therefore the attention circuits, during this period (Amso and Scerif, 2015). Gender can also impact attentional performance and although very few studies have focused on gender differences, girls seem to be more-advantaged (Pascualvaca et al., 1997; Klenberg et al., 2001). Furthermore, abnormal attention development is a symptom of attention deficit hyperactivity disorder (ADHD) in relation to structural and functional brain pathological changes (Biederman and Faraone, 2005). Cross-sectional studies that evaluated the three attentional systems in children with ADHD using the ANT, encountered deficits in the alerting and executive attention networks (Johnson et al., 2008; Gupta and Kar, 2009; Mullane et al., 2011; Casagrande et al., 2012). Specifically, children with ADHD showed lower levels of alertness, consistent with previous theories emphasizing difficulties in arousal regulation in relation to deficits in sustained attention (Russell et al., 2006; Johnson et al., 2007). Furthermore, children with ADHD were more vulnerable to the interference demands, and indeed, response inhibition has been described as the primary deficit in ADHD (Barkley, 1997). To our knowledge, there are no previous longitudinal studies quantifying intra-individual changes over time using the ANT. Prospective longitudinal studies on cognitive function are crucial for understanding typical developmental trajectories, confirming theories of neurodevelopment, and detecting the impact of environmental and social risk factors on cognition in epidemiological studies (Amso and Scerif, 2015; Sunyer et al., 2015; López-Vicente et al., 2016). In contrast to cross-sectional designs, longitudinal studies control for intraindividual differences across time as well as cohort effects and age-related differences in rate of change (Sliwinski and Buschke, 1999; Kraemer et al., 2000). In counterpoint, the magnitude of the practice effects due to repeated testing is a main concern (Dikmen et al., 1999). Ishigami et al. explored the stability, isolability, robustness and reliability of the ANT when administered repeatedly to older adults and patients with multiple sclerosis. Despite some practice effects in alerting and executive control, the network scores were robust 
against practice (Ishigami and Klein, 2011; Ishigami et al., 2013).

We therefore studied the performance of the ANT in a large longitudinal cohort of 7-11 year old children to detect patterns in attention growth. We focused on this age range for several reasons. First, and as mentioned above, some aspects of attention continue to improve throughout middle and late childhood. Second, the feasibility for the administration of the test to the children in their normal class groups (e.g., understanding instructions, ability to work almost autonomously, assuring a prolonged isolation during the execution of the test). Finally, primary school children from 7 years of age were selected in order to increase accuracy of identification of ADHD symptomatology (Applegate et al., 1997). For the current study, in addition to the original three attention networks based on the Posner and Petersen (1990) model, we also analyzed three other measures based on the Mirsky model of attention (Mirsky et al., 1991) and specifically, the Focus component which is related to attentiveness (Egeland and Kovalik-Gran, 2010). For the calculation of these scores we followed the formulas of the Continuous Performance Test (CPT), a widely used computerized measure of different aspects of attention (Conners and Staff, 2000; Oberlin et al., 2005; Adólfsdóttir et al., 2008). We particularly aimed to study the trajectories of attention in a large longitudinal cohort of children using the ANT in a repeated measures design. Furthermore, we examined potential differences between boys and girls, and the role of ADHD symptoms in attention development. Based on the literature, we expect that: (1) the oldest children will show an improvement in alerting; (2) children will show a continued development in executive attention; (3) girls will show an advantage over boys and (4) children with ADHD symptoms will show a delayed developmental pattern in alerting, executive attention and measures related to attentiveness.

\section{MATERIALS AND METHODS}

\section{Participants}

This study is part of the BREATHE (BRain dEvelopment and Air polluTion ultrafine particles in scHool childrEn) project, which aims to assess the association between air pollution in schools and the cognition and behavior of children. The BREATHE project was conducted from January 2012 to March 2013 in 36 schools in Barcelona and 3 in Sant Cugat del Vallès, a smaller city near Barcelona (Catalonia, Spain). The students from these 39 schools in $2 \mathrm{nd}, 3 \mathrm{rd}$, and 4 th primary grades were invited to participate via mail and/or project presentations in the schools. The total number of schoolchildren enrolled for the study was 2,904 (59\% response rate). Seven subjects were excluded from the analysis due to mental, motor or sensory impairment reported by the school. The final sample size at the beginning of the study was of 2,835 children ( $49.6 \%$ girls) aged from 7 to 11 years $(M=8.6$; $S D=0.88)$. The number of participants in session 1 was 2,597 , 2,705 in session 2, 2,621 in session 3, and 2,546 in session 4 .

All parents or legal guardians signed the informed consent approved by the Ethical Committee of the IMIM-Parc Salut Mar.

\section{Instruments}

\section{Neuropsychological Testing}

The tool used to assess the attention domain was the original computerized child ANT (Rueda et al., 2004). In this version, a row of five yellow fish appearing either above or below a fixation point is presented. Children are invited to "feed" the central fish as quickly as possible by pressing either the right or the left arrow key depending on the direction in which the target fish is pointing while ignoring the flanker fish, which point in either the same (congruent) or opposite (incongruent) direction than the middle fish. The target is preceded by visual signals that inform either about the upcoming of the target only (alerting cue) or about the upcoming of the target as well as its location (orienting cue) (Rueda et al., 2012). Each correct answer is followed by a simple animation sequence (the target fish blowing bubbles) and a recorded sound ("woo hoo!"). Incorrect responses are followed by a single tone and no animation of the fish (Rueda et al., 2004).

A session of the ANT consisted of 16 practice trials and four experimental blocks of 32 trials in each (128 trials in total). Each trial represented one of 8 conditions in equal proportions: two Flanker Congruency (congruent and incongruent) $\times$ four Cue Type (no cue, central cue, double cue and spatial cue).

Reaction time (RT) measures of the three attention networks were calculated using RTs associated with a correct response. RTs shorter than $100 \mathrm{~ms}$ were rejected from the RT calculations because of physiological implausibility implying that such a response is perseverative or anticipatory (Conners and Staff, 2000). In addition to alerting, orienting and executive attention networks, three measures related to attentiveness (HRT, HRT$\mathrm{SE}$, and variability) were calculated following the formulas of the CPT (Conners and Staff, 2000). Definition and calculations of the measures are summarized in Table 1. Measures of performance speed were analyzed since they were reported as the most appropriate for assessing change in attentional function in children (Mollica et al., 2005). Lower scores indicate more efficiency in all the measures.

Testing lasted no more than 15 minutes. We followed a strict protocol in order to minimize measurement error: administration of the test was in a quiet and spacious room in the school; children wore headphones in order to avoid noise disturbances; groups were of 10-20 students and there was a trained examiner for every 3-4 children; sufficient distance between children minimized interaction among them; and instructions were always explained following the same structure and by the same examiner. Finally, we also analyzed the influence of environmental factors during task performance, such as day of the week, season, noise, weather, time of the day, and quality of the session (Ballard, 1996). The inclusion of environmental factors as confounders did not change the results, except for alerting (Table S1).

\section{Data Acquisition}

As part of the BREATHE project, a 1-year follow-up with four repeated measures of the attention domain was conducted in the schools, in children in 2nd, 3rd, and 4th grades (aged 710 years at baseline). Most children performed the ANT four times $(76 \%)$. In the cases where an assessment was missed (e.g., 
TABLE 1 | Definitions and calculations of the ANT outcomes.

\begin{tabular}{|c|c|}
\hline Outcomes & Definitions and calculations \\
\hline \multicolumn{2}{|c|}{ ATTENTION NETWORKS } \\
\hline Alerting & RT for No cue - RT for Double Cue trials \\
\hline Orienting & RT for Central Cue - RT for Spatial Cue trials \\
\hline Executive attention & RT for Incongruent - RT for Congruent trials \\
\hline \multicolumn{2}{|c|}{ MEASURES RELATED TO ATTENTIVENESS } \\
\hline HRT & Median RT for correct responses \\
\hline HRT-SE & Standard error of RT for correct responses. Measure of response speed consistency \\
\hline Variability & Standard deviation of the 4 standard error values calculated for each block. Measure of intra-individual variability \\
\hline
\end{tabular}

RT, Reaction Time, SE, Standard Error.

sickness), for the data analysis we considered the temporal order of the school sessions, not the children's "real" attendance to each session. For instance, a child who missed session 2, session 3 was still considered his session 3 . The intervals between the test administrations were on average 3 months. Specifically, the time between session 1 and session 2 was of mean $=2.75$ (1.31) months; mean $=4.54(1.64)$ months between sessions 2 and 3; and mean $=3.91(1.36)$ months between sessions 3 and 4 . The longest interval was between sessions 2 and 3 because of the summer holidays.

\section{Covariates}

Socio-demographic characteristics including child date of birth, gender, maternal education level (primary or less, secondary and university) and home addresses were obtained from a questionnaire completed by parents during 2012. Children's age for each session was calculated based on birth date and session date. A neighborhood socioeconomic status vulnerability index (based on level of education, unemployment, and occupation at the census tract (Sunyer et al., 2015) was calculated at the home address.

Teachers completed an ADHD symptoms questionnaire based on the diagnostic criteria for ADHD as described in the Diagnostic and Statistical Manual of Mental Disorders, fourth Edition (ADHD-DSM-IV; American Psychiatric Association, 2000). ADHD-DSM-IV consists of a list of 18 symptoms categorized under two separate symptom groups (inattention and hyperactivity/impulsivity) with nine symptoms each. Each ADHD symptom is rated on a 4-point scale ( 0 never or rarely, 1 sometimes, 2 often, 3 very often). We recoded options 0 and 1 as 0 (symptom absent), and options 2 and 3 as 1 (symptom present) (Gomez, 2007). We used a categorical variable of ADHD clinical criteria with four categories, according to the presence of 6 or more symptoms of each subtype: no ADHD; ADHD-inattentive; ADHD-hyperactive/impulsive; and ADHDcombined. The teacher ratings of ADHD symptoms used in this study are not to be confounded with a clinical diagnosis of ADHD by a medical doctor.

\section{Statistical Analysis}

Due to the hierarchical structure of the data (children embedded within schools and repeated measures collected on a child over time), we used multilevel mixed-effects linear regression models for each outcome to study the developmental trajectories across sessions. We included random intercepts by school and individual and random slopes by individual for the linear and quadratic effects of age (included to capture the nonlinearity in the growth trajectories of attention, if any). The equation of the model was the following,

$$
\begin{aligned}
\mathrm{Y}_{\text {sit }}= & \left(\beta_{0}+\mathrm{u}_{0 \mathrm{~s}}+\mathrm{s}_{0 \mathrm{i}(\mathrm{s})}\right)+\left(\beta_{1}+\mathrm{s}_{1 \mathrm{i}(\mathrm{s})}\right)^{*}{ }^{*} \mathrm{age}_{\mathrm{sit}} \\
& +\left(\beta_{2}+s_{2 \mathrm{i}(\mathrm{s})}\right)^{*} \mathrm{age}_{\mathrm{sit}}^{2}+\varepsilon_{\mathrm{sit}},
\end{aligned}
$$

Where $\mathrm{Y}_{\text {sit }}$ is the ANT outcome for individual $i$ within school $s$ at session $t, t=\{1,2,3,4\}, \mathrm{u}_{s}$ are random effects at school level, $\mathrm{s}_{\mathrm{i}(\mathrm{s})}$ are random effects associated with the individual $i$ within school $\mathrm{s}$, and $\varepsilon_{\text {sit }}$ are the residuals.

First, random effects associated with age were tested using likelihood-ratio tests. Afterwards, we included the interaction between age and gender, and stratified models were presented if the growth pattern differed according to gender. Then, we tested interactions between age and teacher-rated ADHD symptoms, and the models were stratified when the interactions were statistically significant. Fixed effects were tested using Wald tests. To visualize the shape of the growth function, we plotted the average predicted curve and $95 \%$ confidence band.

Statistical significance was set at $p<0.05$. Statistical analyses were done using R (3.0.2; R Foundation for Statistical Computing) and Stata 12.1 (Stata Corporation, College Station, Texas).

\section{RESULTS}

Children were on average 8.6 years old at baseline and $49.6 \%$ were girls. Maternal education level was high (58.9\% of mothers had a university degree). According to the questionnaires rated by the teachers $10.5 \%$ of children presented ADHD symptoms, being the inattentive subtype the most prevalent across age groups (Table 2).

In the multilevel mixed-effects linear regression models we found improvements related to age for all the outcomes, except orienting. Gender interacted with age in executive attention $(p$ for interaction $<0.001$ ) and HRT ( $p$ for interaction $<0.001$ ). Teacher-rated ADHD symptoms interacted with age in executive 
TABLE 2 | Demographic and clinical characteristics of the sample by grade $(n=2835)$.

\begin{tabular}{|c|c|c|c|}
\hline & 2 nd grade $(n=1064)$ & 3rd grade $(n=1015)$ & 4th grade $(n=756)$ \\
\hline Age at session 1 (mean, SD) & $7.7(0.33)$ & $8.7(0.37)$ & $9.7(0.36)$ \\
\hline Gender (\% girls) & 49.0 & 48.9 & 51.5 \\
\hline Home socioeconomic vulnerability index (mean, SD) & $0.45(0.20)$ & $0.45(0.21)$ & $0.45(0.21)$ \\
\hline \multicolumn{4}{|l|}{ Maternal education (\%) } \\
\hline Primary or less & 15.2 & 11.0 & 11.8 \\
\hline \multicolumn{4}{|l|}{ ADHD subtypes -DSM-IV, teachers form ${ }^{\mathrm{a}}(\%)$} \\
\hline No-ADHD & 90.0 & 90.6 & 88.0 \\
\hline ADHD-inattentive & 6.13 & 6.18 & 6.41 \\
\hline ADHD-hyperactive/impulsive & 1.1 & 1.6 & 2.5 \\
\hline
\end{tabular}

$A D H D$, Attention Deficit Hyperactivity Disorder.

${ }^{a} A D H D$ Criteria of Diagnostic and Statistical Manual of Mental Disorders, fourth edition. Teachers form.

attention $(p$ for interaction $=0.027)$, HRT $(p$ for interaction $=$ 0.014 ) and variability ( $p$ for interaction $=0.009$ ) (Table 3$)$.

The inclusion of maternal education and socioeconomic status in the models did not change the results (Table S2). Furthermore, the interactions between age and maternal education ( $p$ for interaction $=0.263$ ) and socioeconomic status ( $p$ for interaction $=0.093$ ) were unrelated to executive attention development (Table S3). Figures 1-6 represent age-associated changes in the ANT networks scores and measures related to attentiveness.

Regarding the network scores, for alerting we detected a quadratic curve indicating a further growth for the oldest ages (Figure 1, Table 3). Different age-related patterns by gender or ADHD symptoms were not found. We obtained no significant age effect for orienting (Figure 1, Table 3). For executive attention, girls performed better at young ages. However, males showed a faster cognitive growth and reached girls performance at around 10 years of age (Figure 1). Children with teacher-rated ADHD symptoms, specifically the inattentive and combined subtypes, performed worse than children with no ADHD symptoms until age 9 in executive attention. No age-related changes were encountered in children with ADHD hyperactiveimpulsive symptoms (Figure 2, Table 3).

Regarding measures related to attentiveness, for HRT quadratic curves were found indicating more pronounced increases in performance in the younger groups (Table 3). Although boys were significantly quicker, girls showed a more pronounced improvement during the age range studied and reached the levels of boys at age 11 (Figure 3). Children with ADHD symptoms showed quicker HRT across the entire age range but without reaching the levels of children with no ADHD symptoms (Figure 4, Table 3). For HRT-SE, we found a quadratic curve, indicating a greater improvement in the younger groups that stabilized at around 10 years of age (Figure 5). Different age-related patterns by gender or ADHD symptoms were not detected. Finally, for variability different age-related patterns by gender were not detected. Children with
ADHD hyperactive-impulsive and combined symptoms showed higher variability than those without ADHD symptoms across all age groups (Table 3, Figure 6). No age-related changes were encountered in children with ADHD inattention symptoms (Table 3).

\section{DISCUSSION}

The present study explored, for the first time, the attention development using the ANT in a large sample $(n=2,835)$ of primary-school children in a prospective longitudinal study using repeated measures. The pathways of the three attention networks and measures related to attentiveness showed different developmental trajectories. Specifically, the attention development in the age range studied was more pronounced in executive attention, HRT, HRT-SE, and variability, particularly from 7 to 9 years of age. We detected gender differences in executive attention and HRT up to age 10. Children with teacher-rated ADHD symptoms showed a developmental delay in executive attention, HRT and variability.

The trajectories of attention growth observed confirm previous cross-sectional studies that used the ANT, which described an ongoing development of some aspects of attention during early and late childhood (i.e., ages 5-12) (Mezzacappa, 2004; Rueda et al., 2004; Mullane et al., 2016; Federico et al., 2017). This is consistent with neuroimaging studies reporting continued myelinization of the neural circuitry involved in attention processes until adolescence (Hudspeth and Pribram, 1992; Konrad et al., 2005). Furthermore, larger improvements in the younger groups correlate with the fourth rapid brain growth stage (i.e., ages between 6 and 8 years; Epstein, 1986).

Regarding the developmental trajectories of the attention networks, for alerting we found a slow progression toward the end of the investigated age range (Ridderinkhof et al., 1997; Gupta and Kar, 2009; Mullane et al., 2016; Federico et al., 2017). Alertness starts to develop in the first few postnatal weeks and 
TABLE 3 | Age-associated changes (coefficient, $95 \% \mathrm{Cl})^{\dagger}$ in the ANT outcomes during the 1-year follow-up.

\begin{tabular}{|c|c|c|c|c|}
\hline Outcome & Age & $p$ & $\operatorname{Age}^{2 \|}$ & $p$ \\
\hline \multicolumn{5}{|l|}{ ALERTING } \\
\hline All & $4.65(-1.26,10.57)$ & 0.123 & $-1.44(-2.84,-0.04)$ & 0.043 \\
\hline \multicolumn{5}{|l|}{ ORIENTING } \\
\hline All & $-1.47(-2.96,0.02)$ & 0.053 & - & \\
\hline \multicolumn{5}{|l|}{ EXECUTIVE ATTENTION } \\
\hline No ADHD & $-6.02(-7.28,-4.77)$ & $<0.001$ & - & \\
\hline ADHD-inattentive & $-10.69(-16.69,-4.68)$ & $<0.001$ & - & \\
\hline ADHD-hyperactive-impulsive & $-3.89(-13.44,5.64)$ & 0.423 & - & \\
\hline ADHD-combined & $-16.55(-29.20,-3.91)$ & 0.010 & - & \\
\hline \multicolumn{5}{|l|}{ HRT } \\
\hline ADHD-inattentive & $-197.90(-240.19,-155.61)$ & $<0.001$ & $22.16(12.52,31.80)$ & $<0.001$ \\
\hline ADHD-hyperactive-impulsive & $-58.24(-80.30,-36.18)$ & $<0.001$ & - & \\
\hline ADHD-combined & $-79.96(-104.23,-55.68)$ & $<0.001$ & - & \\
\hline \multicolumn{5}{|l|}{ HRT-SE } \\
\hline All & $-62.26(-69.44,-55.09)$ & $<0.001$ & $7.50(5.82,9.18)$ & $<0.001$ \\
\hline \multicolumn{5}{|l|}{ VARIABILITY } \\
\hline No ADHD & $-2.93(-3.86,-2.01)$ & $<0.001$ & - & \\
\hline ADHD-inattentive & $1.24(-2.12,4.60)$ & 0.469 & - & \\
\hline ADHD-hyperactive-impulsive & $-8.83(-15.65,-2.01)$ & 0.011 & - & \\
\hline ADHD-combined & $-6.88(-13.28,-0.49)$ & 0.035 & - & \\
\hline
\end{tabular}

Cl, Confidence Interval, RT, Reaction Time, SE, Standard Error, ADHD, Attention Deficit and Hyperactivity Disorder.

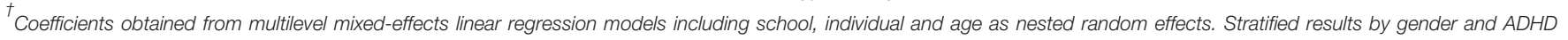
symptoms are provided when $p$-value for interaction $\leq 0.05$.

"When the association with age was not linear, a quadratic function was fitted.

-No effect.

"All" refers to all children.

by the third month babies are able to maintain an alert state in relation to the external sensory stimulation (Rueda et al., 2015). Despite the presence in infancy, the alerting network undergoes a significant improvement during late childhood in relation to continued development of frontal regions during this period (Rueda et al., 2004). The orienting network starts developing very early in infancy; newborns show head an eye movements toward a peripheral cue and from age 4 months there is great stability (Clohessy et al., 2001; Rueda et al., 2004; Gupta and Kar, 2009). For orienting we obtained no significant age changes in children from 7 to 11 years old, consistent with the formerly described early maturation of this network. The executive attention network follows a more prolonged development during childhood and into early adolescence (Ridderinkhof et al., 1997; Rueda et al., 2004; van Meel et al., 2012; Loher and Roebers, 2013; Mullane et al., 2016). Executive attention involves detecting and resolving conflict among responses, error detection and response inhibition. Its development is related to the maturation of the anterior cingulate cortex and lateral prefrontal cortex (Bush et al., 2000) which are not fully matured until adolescence
(Romine and Reynolds, 2005). Furthermore, we encountered that the developmental course of the executive attention network differed by gender. Girls were superior compared to boys in the younger groups, probably reflecting variations in the maturation rate between males and females up to ages of 9 and 11 years (Pascualvaca et al., 1997). A more rapid biological, cognitive, and social-emotional development of girls is in fact well-known (Keenan and Shaw, 1997; Cahill, 2006). Finally, in our study the executive attention trajectory was unrelated to maternal education or socioeconomic status, in contrast to previous literature documenting an association between different indicators of socioeconomic status and executive function development (Hackman and Farah, 2009).

Regarding the measures related to attentiveness, significant improvements in reaction time (HRT), response speed consistency (HRT-SE) and intra-individual variability were found over the age range studied, and particularly between ages 7 and 9 years (Levy, 1980; Rebok et al., 1997). Boys had faster reaction times compared to girls (Pascualvaca et al., 1997; Klenberg et al., 2001), although the progression was further for 


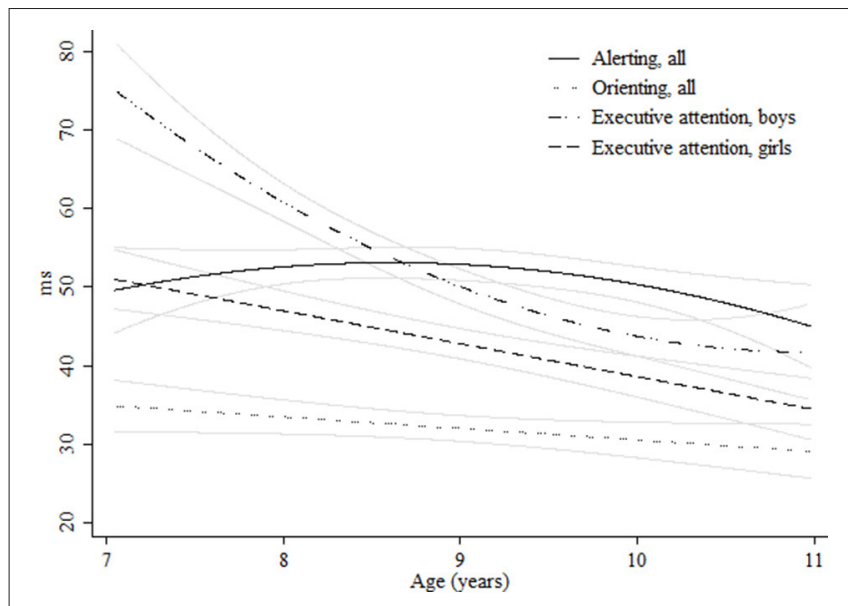

FIGURE 1 | Age-associated predicted curves for the network scores. Average predicted curves and $95 \%$ confidence bands. Stratified results by gender are provided for executive attention.

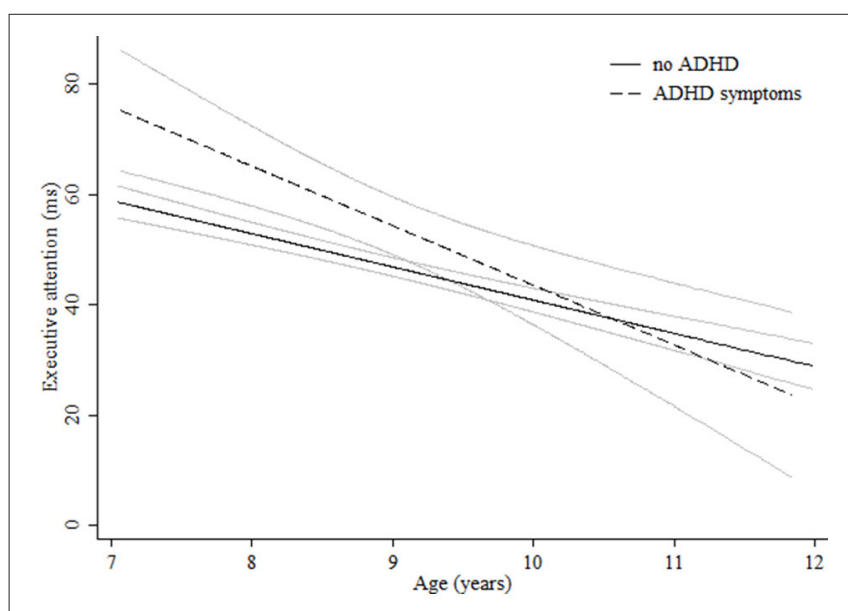

FIGURE 2 | Age-associated predicted curves for executive attention. Average predicted curves and $95 \%$ confidence bands. Stratified results by ADHD symptoms are provided.

females. The lack of significant differences in the trajectories of response speed consistency and intra-individual variability by gender, suggests similar age trends for males and females for these aspects of attention (Pascualvaca et al., 1997).

The developmental trajectories for executive attention, response speed and intra-individual variability were significantly different in children with teacher-rated ADHD symptoms compared to typically developing children. Neuroimaging studies have reported a delay of 2-3 years in brain maturation in $\mathrm{ADHD}$, and not a complete deviation from typical development (Castellanos et al., 2002; Shaw et al., 2007). Difficulties in executive attention and alerting networks have been described as deficits that underlie ADHD (Berger and Posner, 2000). As expected, typically developing children outperformed children with ADHD symptoms in the executive attention network which involves response inhibition, indeed the essential impairment

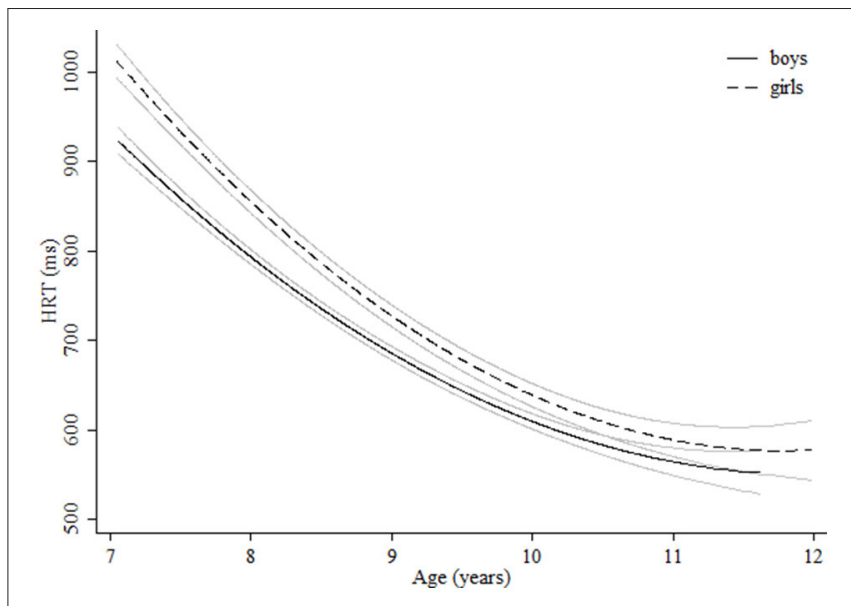

FIGURE 3 | Age-associated predicted curves for HRT. Average predicted curves and 95\% confidence bands. Stratified results by gender are provided.

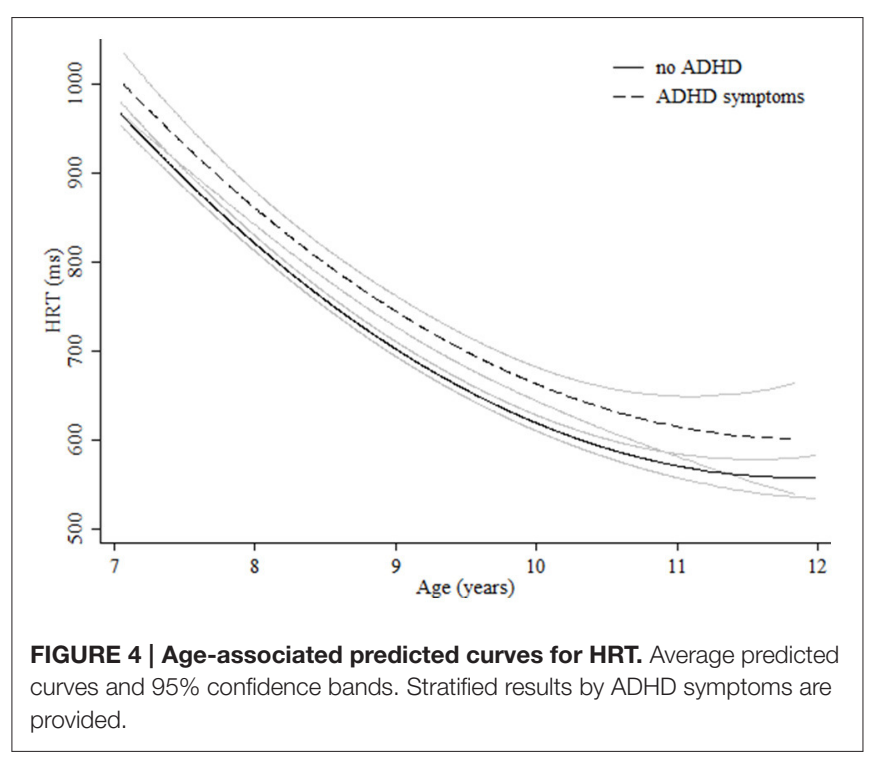

in this disorder (Barkley, 1997). However, we encountered no differences in the developmental trajectory of alerting in the age range studied between the two groups. The late further development of the alerting network (e.g., from 10 years of age) (Ridderinkhof et al., 1997; Rueda et al., 2004) may partly explain these results. Furthermore, in the published literature there are also discrepancies related to a weaker alertness in ADHD (Booth et al., 2007; Adólfsdóttir et al., 2008; Johnson et al., 2008; Gupta and Kar, 2009; Mullane et al., 2011; Casagrande et al., 2012). Regarding reaction time in responding to a target, slower response speed has been reported previously for children with ADHD (Gupta and Kar, 2009; Mullane et al., 2011), and that is in fact what we observed in our study. Children with ADHD symptoms had a developmental trajectory for response speed that paralleled the growth curve for typically developing children but on a lower track. Finally, in relation to intra-individual 


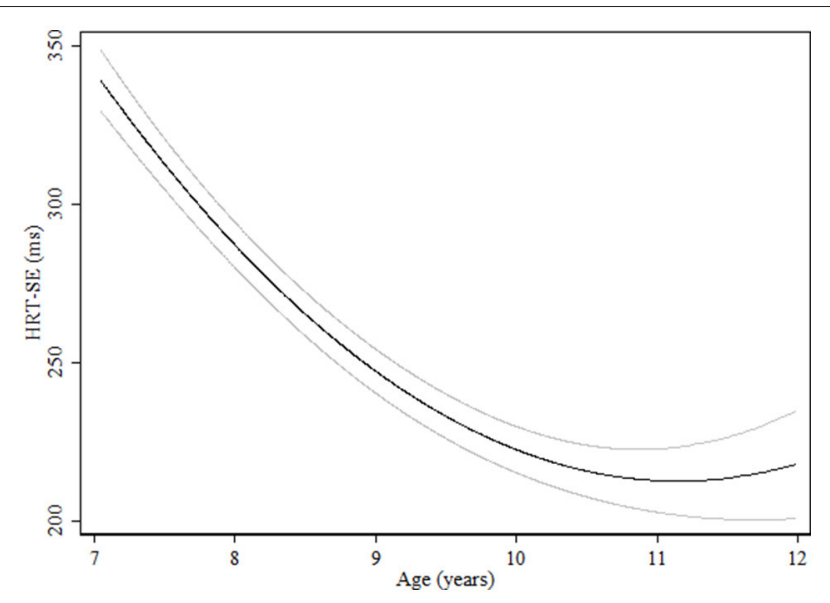

FIGURE 5 | Age-associated predicted curve for HRT-SE. Average predicted curve and 95\% confidence band.

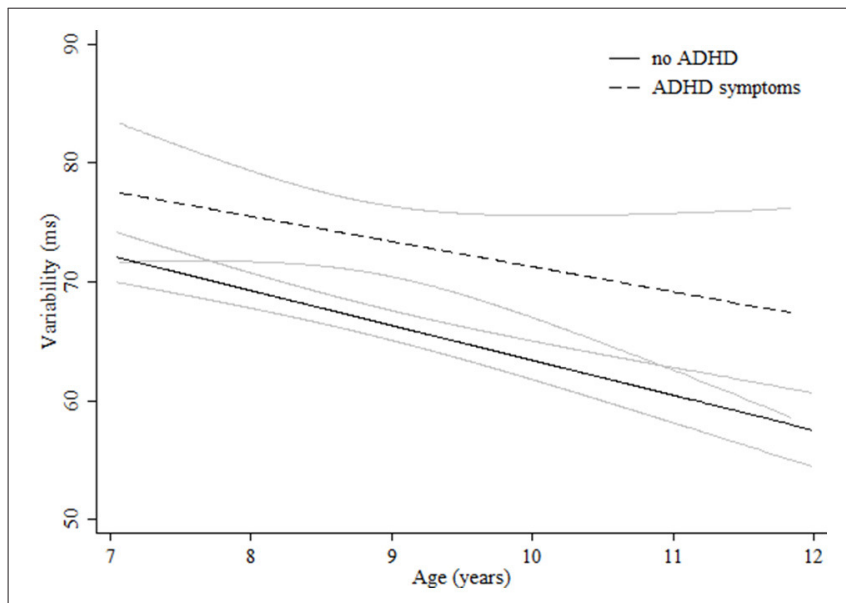

FIGURE 6 | Age-associated predicted curves for Variability. Average predicted curves and $95 \%$ confidence bands. Stratified results by ADHD symptoms are provided.

variability, we encountered more variability in response speed consistency in children with ADHD symptoms compared to children without ADHD symptoms. The moment-to-moment fluctuations in attention is in fact the most remarkable symptom of ADHD (Castellanos and Tannock, 2002). Indeed, intraindividual response variability measures, defined as short-term fluctuations in performance of an individual over a time-scales of seconds, have been described as the best to discriminate between ADHD and control groups (Russell et al., 2006). Finally, in relation to ADHD subtypes, no clear differential patterns of attention trajectories were observed despite the well-described clinical differences between them (Cantwell and Baker, 1992).

This study has some strengths and limitations that need to be considered. The present findings are dependent on the version of the ANT used, the age range of the participants and the teacherrated $\mathrm{ADHD}$ symptoms - not a medical diagnosis of ADHD. However, we found that the child ANT (Rueda et al., 2004) was able to detect attention trajectories in childhood, including gender differences and ADHD symptoms. In relation to the age effects, the late development of the alerting network or the earlier executive attention development in females, for instance, still needs further investigation. Furthermore, the normalization of the performance in executive attention at around 10 years of age in children with teacher-rated ADHD symptoms needs future confirmation. Further studies using the original ANT (Fan et al., 2002) are warranted in order to discard any ceiling effects of the child ANT in late childhood. Finally, the cognitive change observed with repeated test application over a 1-year period may still include some practice effects. However, in previous studies, despite short test-retest intervals (i.e., hours or days) in attentional function in children or in adults the magnitude of the practice effects was moderate to small and the ANT networks showed robustness against practice (Mollica et al., 2005; Ishigami and Klein, 2011). Furthermore, the exact age in each assessment and the time intervals between the neuropsychological testing varied among the children and that contributed to minimizing practice effects. Strengths of this population-based study include the large sample size and the longitudinal design for the detection of cognitive change. Repeated measurements withinparticipants provided the prospective data required to define developmental trajectories. In addition to the attention networks of the ANT, three measures related to attentiveness were also calculated and allowed us to explore the growth patterns of more aspects of attention. Furthermore, we analyzed the role of gender and ADHD symptoms in attention development. Finally, the study of the attention development in gradeschoolers can be used to identify students who deviate from normality and the identification of possible environmental or social risk factors. This would contribute to the implementation of school accommodations or other interventions needed for these children. The implications of attentional deficits in the school setting are non-negligible since they are associated with behavioral difficulties and poor academic achievement (e.g., lack of self-control, failure to complete tasks, and commission of procedural mistakes) (Pascualvaca et al., 1997; Anderson, 2002).

In summary, we observed an ongoing development of some aspects of attention in primary school aged children. Nevertheless, the developmental changes were more evident in executive attention and measures related to attentiveness, and in the younger groups. Furthermore, girls were more advantaged at younger ages and children with teacher-rated ADHD symptoms showed a delayed development in some attention processes. Our findings support the ANT for assessing attention in children in large epidemiological studies.

\section{AUTHOR CONTRIBUTIONS}

ES, JF, and JS conceptualized and designed the study and drafted the initial manuscript. RG, ME, and XB supported and supervised the statistical analyses and revised the manuscript. MA coordinated and supervised data collections and critically reviewed the manuscript. ML, AL, JJ, and AC supervised the interpretation of the results and critically reviewed the manuscript. 


\section{FUNDING}

The research leading to these results received funding from the European Research Council under the ERC Grant Agreement number 268479 - the BREATHE project. JJ holds a Miguel Servet contract (MS14/00108) awarded by the Spanish Institute of Health Carlos III (Ministry of Economy and Competitiveness).

\section{ACKNOWLEDGMENTS}

We want to thank all the children and their families who participated in the study for their altruism and particularly to the schools Antoni Brusi, Baloo, Betània - Patmos, Centre d'estudis Montseny, Col-legi Shalom, Costa i Llobera, El sagrer, Els Llorers, Escola Pia de Sarrià, Escola Pia Balmes, Escola concertada Ramon Llull, Escola Nostra Sra. de Lourdes, Escola Tècnica Professional

\section{REFERENCES}

Adólfsdóttir, S., Sørensen, L., and Lundervold, A. J. (2008). The attention network test: a characteristic pattern of deficits in children with ADHD. Behav. Brain Functions 4:9. doi: 10.1186/1744-9081-4-9

American Psychiatric Association (2000). Diagnostic and Statistical Manual of Mental Disorders, 4th Edn. DSM-IV-TR ${ }^{\circledR}$. Washington, DC: American Psychiatric Publishing.

Amso, D., and Johnson, S. P. (2006). Learning by selection: visual search and object perception in young infants. Dev. Psychol. 42, 1236-1245. doi: 10.1037/0012-1649.42.6.1236

Amso, D., and Scerif, G. (2015). The attentive brain: insights from developmental cognitive neuroscience. Nat. Rev.. Neurosci. 16, 606-619. doi: 10.1038/nrn4025

Anderson, P. (2002). Assessment and development of executive function (EF) during childhood. Child Neuropsychol. 8, 71-82. doi: 10.1076/chin.8.2.71.8724

Applegate, B., Lahey, B. B., Hart, E. L., Biederman, J., Hynd, G. W., Barkley, R. A., et al. (1997). Validity of the age-of-onset criterion for ADHD: a report from the DSM-IV field trials. J. Am. Acad. Child Adolesc. Psychiatry 36, 1211-1221.

Ballard, J. C. (1996). Computerized assessment of sustained attention: a review of factors affecting vigilance performance. J. Clin. Exp. Neuropsychol. 18, 843-863. doi: 10.1080/01688639608408307

Barkley, R. A. (1997). Behavioral inhibition, sustained attention, and executive functions: constructing a unifying theory of ADHD. Psychol. Bull. 121, 65-94.

Berger, A., and Posner, M. I. (2000). Pathologies of brain attentional networks. Neurosci. Biobehav. Rev. 24, 3-5. doi: 10.1016/s0149-7634(99)00046-9

Biederman, J., and Faraone, S. V. (2005). Attention-deficit hyperactivity disorder. Lancet 366, 237-248. doi: 10.1016/S0140-6736(05)66915-2

Booth, J. E., Carlson, C. L., and Tucker, D. M. (2007). Performance on a neurocognitive measure of alerting differentiates ADHD combined and inattentive subtypes: a preliminary report. Arch. Clin. Neuropsychol. 22, 423-432. doi: 10.1016/j.acn.2007.01.017

Bush, G., Luu, P., and Posner, M. I. (2000). Cognitive and emotional influences in anterior cingulate cortex. Trends Cogn. Sci. 4, 215-222. doi: 10.1016/s1364-6613(00)01483-2

Cahill, L. (2006). Why sex matters for neuroscience. Nat. Rev.. Neurosci. 7, 477-484. doi: 10.1038/nrn1909

Cantwell, D. P., and Baker, L. (1992). Attention deficit disorder with and without hyperactivity: a review and comparison of matched groups. J. Am. Acad. Child Adolesc. Psychiatry 31, 432-438. doi: 10.1097/00004583-199205000-00008

Casagrande, M., Martella, D., Ruggiero, M. C., Maccari, L., Paloscia, C., Rosa, C., et al. (2012). Assessing attentional systems in children with Attention Deficit Hyperactivity Disorder. Arch. Clin. Neuropsychol. 27, 30-44. doi: 10.1093/arclin/acr085

Castellanos, F. X., Lee, P. P., Sharp, W., Jeffries, N. O., Greenstein, D. K., Clasen, L. S., et al. (2002). Developmental trajectories of brain volume abnormalities in del Clot, Ferran i Clua, Francesc Macià, Frederic Mistral, Infant Jesús, Joan Maragall, Jovellanos, La Llacuna del Poblenou, Lloret, Menéndez Pidal, Nuestra Señora del Rosario, Miralletes, Ramon Llull, Rius i Taulet, Pau Vila, Pere Vila, Pi d'en Xandri, Projecte, Prosperitat, Sant Ramon Nonat - Sagrat Cor, Santa Anna, Sant Gregori, Sagrat Cor Diputació, Tres Pins, Tomàs Moro, Torrent d'en Melis, Virolai. We also acknowledge: Xavier Mayoral for developing the computerized ANT; Laura Bouso, Judit González, Pere Figueras, and Cecilia Persavento for the fieldwork; Núria Sebastián for psychological assessment. ISGlobal is a member of the CERCA Programme, Generalitat de Catalunya.

\section{SUPPLEMENTARY MATERIAL}

The Supplementary Material for this article can be found online at: http://journal.frontiersin.org/article/10.3389/fpsyg. 2017.00655/full\#supplementary-material

children and adolescents with attention-deficit/hyperactivity disorder. JAMA 288, 1740-1748. doi: 10.1001/jama.288.14.1740

Castellanos, F. X., and Tannock, R. (2002). Neuroscience of attentiondeficit/hyperactivity disorder: the search for endophenotypes. Nat. Rev. Neurosci. 3, 617-628. doi: 10.1038/nrn896

Clohessy, A. B., Posner, M. I., and Rothbart, M. K. (2001). Development of the functional visual field. Acta Psychol. 106, 51-68. doi: 10.1016/S0001-6918(00)00026-3

Conners, C. K., and Staff, M. H. S. (2000). Conners' Continuous Performance Test II: Computer Program for Windows Technical Guide and Software Manual. North Tonwanda, NY: Multi-Health Systems.

Corbetta, M., Akbudak, E., Conturo, T. E., Snyder, A. Z., Ollinger, J. M., Drury, H. A., et al. (1998). A common network of functional areas for attention and eye movements. Neuron 21, 761-773.

Dikmen, S. S., Heaton, R. K., Grant, I., and Temkin, N. R. (1999). Test-retest reliability and practice effects of expanded Halstead-Reitan Neuropsychological Test Battery. J. Int. Neuropsychol. Soc. 5, 346-356.

Egeland, J., and Kovalik-Gran, I. (2010). Validity of the factor structure of Conners' CPT. J. Atten. Disord. 13, 347-357. doi: 10.1177/1087054709332477

Epstein, H. T. (1986). Stages in human brain development. Brain Res. 395, 114-119. Eriksen, B. A., and Eriksen, C. W. (1974). Effects of noise letters upon the identification of a target letter in a nonsearch task. Percept. Psychophys. 16, 143-149.

Fan, J., McCandliss, B. D., Fossella, J., Flombaum, J. I., and Posner, M. I. (2005). The activation of attentional networks. Neuroimage 26, 471-479. doi: 10.1016/j.neuroimage.2005.02.004

Fan, J., McCandliss, B. D., Sommer, T., Raz, A., and Posner, M. I. (2002). Testing the efficiency and independence of attentional networks. J. Cogn. Neurosci. 14, 340-347. doi: 10.1162/089892902317361886

Federico, F., Marotta, A., Martella, D., and Casagrande, M. (2017). Development in attention functions and social processing: evidence from the attention network test. Br. J. Dev. Psychol. 35, 169-185. doi: 10.1111/bjdp.12154

Forns, J., Esnaola, M., López-Vicente, M., Suades-González, E., AlvararezPedrerol, M., Julvez, J., et al. (2014). The n-back test and the attentional network task as measures of child neuropsychological development in epidemiological studies. Neuropsychology 28, 519-529. doi: 10.1037/neu 0000085

Gomez, R. (2007). Testing gender differential item functioning for ordinal and binary scored parent rated ADHD symptoms. Pers. Individ. Dif. 42, 733-742. doi: 10.1016/j.paid.2006.08.011

Gupta, R., and Kar, B. R. (2009). Development of attentional processes in ADHD and normal children. Prog. Brain Res. 176, 259-276. doi: 10.1016/S0079-6123(09)17614-8

Hackman, D. A., and Farah, M. J. (2009). Socioeconomic status and the developing brain. Trends Cogn. Sci. 13, 65-73. doi: 10.1016/j.tics.2008.11.003 
Hudspeth, W. J., and Pribram, K. H. (1992). Psychophysiological indices of cerebral maturation. Int. J. Psychophysiol. 12, 19-29.

Ishigami, Y., Fisk, J. D., Wojtowicz, M., and Klein, R. M. (2013). Repeated measurement of the attention components of patients with multiple sclerosis using the Attention Network Test-Interaction (ANT-I): stability, isolability, robustness, and reliability. J. Neurosci. Methods 216, 1-9. doi: 10.1016/j.jneumeth.2013.02.013

Ishigami, Y., and Klein, R. M. (2011). Repeated measurement of the components of attention of older adults using the two versions of the attention network test: stability, isolability, robustness, and reliability. Front. Aging Neurosci. 3:17. doi: 10.3389/fnagi.2011.00017

Johnson, K. A., Kelly, S. P., Bellgrove, M. A., Barry, E., Cox, M., Gill, M., et al. (2007). Response variability in attention deficit hyperactivity disorder: evidence for neuropsychological heterogeneity. Neuropsychologia 45, 630-638. doi: 10.1016/j.neuropsychologia.2006.03.034

Johnson, K. A., Robertson, I. H., Barry, E., Mulligan, A., Dáibhis, A., Daly, M., et al. (2008). Impaired conflict resolution and alerting in children with ADHD: evidence from the Attention Network Task (ANT). J. Child Psychol. Psychiatry 49, 1339-1347. doi: 10.1111/j.1469-7610.2008.01936.x

Keenan, K., and Shaw, D. (1997). Developmental and social influences on young girls' early problem behavior. Psychol. Bull. 121, 95-113.

Klenberg, L., Korkman, M., and Lahti-Nuuttila, P. (2001). Differential development of attention and executive functions in 3- to 12-year-old Finnish children. Dev. Neuropsychol. 20, 407-428. doi: 10.1207/S15326942DN2001_6

Konrad, K., Neufang, S., Thiel, C. M., Specht, K., Hanisch, C., Fan, J., et al. (2005). Development of attentional networks: an fMRI study with children and adults. Neuroimage 28, 429-439. doi: 10.1016/j.neuroimage.2005. 06.065

Kraemer, H. C., Yesavage, J. A., Taylor, J. L., and Kupfer, D. (2000). How can we learn about developmental processes from cross-sectional studies, or can we? Am. J. Psychiatry 157, 163-171. doi: 10.1176/appi.ajp.157.2.163

Levy, F. (1980). The development of sustained attention (vigilance) and inhibition in children: some normative data. J. Child Psychol. Psychiatry 21, 77-84.

Loher, S., and Roebers, C. M. (2013). Executive functions and their differential contribution to sustained attention in 5-to 8-Year-Old Children. J. Educ. Dev. Psychol. 3, 51-63. doi: 10.5539/jedp.v3n1p51

López-Vicente, M., Forns, J., Suades-González, E., Esnaola, M., GarcíaEsteban, R., Álvarez-Pedrerol, M., et al. (2016). Developmental trajectories in primary schoolchildren using n-back task. Front. Psychol. 7:716. doi: $10.3389 /$ fpsyg.2016.00716

Mezzacappa, E. (2004). Alerting, orienting, and executive attention: developmental properties and sociodemographic correlates in an epidemiological sample of young, urban children. Child Dev. 75, 1373-1386. doi: $10.1111 / j .1467-8624.2004 .00746 . x$

Mirsky, A. F., Anthony, B. J., Duncan, C. C., Ahearn, M. B., and Kellam, S. G. (1991). Analysis of the elements of attention: a neuropsychological approach. Neuropsychol. Rev. 2, 109-145.

Mollica, C. M., Maruff, P., Collie, A., and Vance, A. (2005). Repeated assessment of cognition in children and the measurement of performance change. Child Neuropsychol. 11, 303-310. doi: 10.1080/092970490911306

Mullane, J. C., Corkum, P. V., Klein, R. M., McLaughlin, E. N., and Lawrence, M. A. (2011). Alerting, orienting, and executive attention in children with ADHD. J. Atten. Disord. 15, 310-320. doi: 10.1177/1087054710366384

Mullane, J. C., Lawrence, M. A., Corkum, P. V., Klein, R. M., and McLaughlin, E. N. (2016). The development of and interaction among alerting, orienting, and executive attention in children. Child Neuropsychol. 22, 155-176. doi: 10.1080/09297049.2014.981252

Oberlin, B. G., Alford, J. L., and Marrocco, R. T. (2005). Normal attention orienting but abnormal stimulus alerting and conflict effect in combined subtype of ADHD. Behav. Brain Res. 165, 1-11. doi: 10.1016/j.bbr.2005.06.041

Pascualvaca, D. M., Anthony, B. J., Arnold, L. E., Rebok, G. W., Ahearn, M. B., Kellam, S. G., et al. (1997). Attention performance in an epidemiological sample of urban children: the role of gender and verbal intelligence. Child Neuropsychol. 3, 13-27. doi: 10.1080/09297049708401365

Petersen, S. E., and Posner, M. I. (2012). The attention system of the human brain: 20 years after. Annu. Rev. Neurosci. 35, 73-89. doi: 10.1146/annurev-neuro-062111-150525

Posner, M. I., and Petersen, S. E. (1990). The attention system of the human brain. Annu. Rev. Neurosci. 13, 25-42. doi: 10.1146/annurev.ne.13.030190.000325
Posner, M. I., and Rothbart, M. K. (2007). Research on attention networks as a model for the integration of psychological science. Annu. Rev. Psychol. 58, 1-23. doi: 10.1146/annurev.psych.58.110405.085516

Posner, M. I., Sheese, B. E., Odludaş, Y., and Tang, Y. (2006). Analyzing and shaping human attentional networks. Neural Netw. 19, 1422-1429. doi: 10.1016/j.neunet.2006.08.004

Rebok, G. W., Smith, C. B., Pascualvaca, D. M., Mirsky, A. F., Anthony, B. J., and Kellam, S. G. (1997). Developmental changes in attentional performance in urban children from eight to thirteen years. Child Neuropsychol. 3, 28-46. doi: 10.1080/09297049708401366

Rice, D., and Barone, S. (2000). Critical periods of vulnerability for the developing nervous system: evidence from humans and animal models. Environ. Health Perspect. 108, 511-533. doi: 10.1289/ehp.00108s3511

Ridderinkhof, K. R., van der Molen, M. W., Band, G. P., and Bashore, T. R. (1997). Sources of interference from irrelevant information: a developmental study. J. Exp. Child Psychol. 65, 315-341. doi: 10.1006/jecp.1997.2367

Romine, C. B., and Reynolds, C. R. (2005). A model of the development of frontal lobe functioning: findings from a meta-analysis. Appl. Neuropsychol. 12, 190-201. doi: 10.1207/s15324826an1204_2

Rueda, M., Rosario, Pozuelos Joan, P., and Cómbita Lina, M. (2015). Cognitive Neuroscience of Attention. Available online at: https://www.researchgate.net/ profile/Maria_Rueda/publication/282734934_Cognitive_Neuroscience_of_ Attention_From_brain_mechanisms_to_individual_differences_in_efficiency/ links/561ac29608ae78721f9f8d30.pdf

Rueda, M. R., Checa, P., and Cómbita, L. M. (2012). Enhanced efficiency of the executive attention network after training in preschool children: immediate changes and effects after two months. Dev. Cogn. Neurosci. 2 (Suppl. 1), S192-S204. doi: 10.1016/j.dcn.2011.09.004

Rueda, M. R., Fan, J., McCandliss, B. D., Halparin, J. D., Gruber, D. B., Lercari, L. P., et al. (2004). Development of attentional networks in childhood. Neuropsychologia 42, 1029-1040. doi: 10.1016/j.neuropsychologia.2003.12.012

Russell, V. A., Oades, R. D., Tannock, R., Killeen, P. R., Auerbach, J. G., Johansen, E. B., et al. (2006). Response variability in Attention-Deficit/Hyperactivity Disorder: a neuronal and glial energetics hypothesis. Behav. Brain Funct. 2:30. doi: 10.1186/1744-9081-2-30

Shaw, P., Eckstrand, K., Sharp, W., Blumenthal, J., Lerch, J. P., Greenstein, D., et al. (2007). Attention-deficit/hyperactivity disorder is characterized by a delay in cortical maturation. Proc. Natl. Acad. Sci. U.S.A. 104, 19649-19654. doi: 10.1073/pnas.0707741104

Sliwinski, M., and Buschke, H. (1999). Cross-sectional and longitudinal relationships among age, cognition, and processing speed. Psychol. Aging 14, $18-33$.

Spira, E. G., and Fischel, J. E. (2005). The impact of preschool inattention, hyperactivity, and impulsivity on social and academic development: a review. J. Child Psychol. Psychiatry 46, 755-773. doi: 10.1111/j.1469-7610.2005.01466.x

Sunyer, J., Esnaola, M., Alvarez-Pedrerol, M., Forns, J., Rivas, I., López-Vicente, M., et al. (2015). Association between traffic-related air pollution in schools and cognitive development in primary school children: a prospective cohort study. PLoS Med. 12:e1001792. doi: 10.1371/journal.pmed.1001792

van Meel, C. S., Heslenfeld, D. J., Rommelse, N. N., Oosterlaan, J., and Sergeant, J. A. (2012). Developmental trajectories of neural mechanisms supporting conflict and error processing in middle childhood. Dev. Neuropsychol. 37, 358-378. doi: 10.1080/87565641.2011.653062

Welsh, M. C., and Pennington, B. F. (1988). Assessing frontal lobe functioning in children: views from developmental psychology. Dev. Neuropsychol. 4, 199-230. doi: 10.1080/87565648809540405

Conflict of Interest Statement: The authors declare that the research was conducted in the absence of any commercial or financial relationships that could be construed as a potential conflict of interest.

Copyright $\odot 2017$ Suades-González, Forns, García-Esteban, López-Vicente, Esnaola, Álvarez-Pedrerol, Julvez, Cáceres, Basagaña, López-Sala and Sunyer. This is an open-access article distributed under the terms of the Creative Commons Attribution License (CC BY). The use, distribution or reproduction in other forums is permitted, provided the original author(s) or licensor are credited and that the original publication in this journal is cited, in accordance with accepted academic practice. No use, distribution or reproduction is permitted which does not comply with these terms. 